\title{
A new imaging method: optofluidic microscopy
}

\section{Xin Heng, David Erickson, Demetri Psaltis, Changhuei Yang}

Xin Heng, David Erickson, Demetri Psaltis, Changhuei Yang, "A new imaging method: optofluidic microscopy," Proc. SPIE 6003, Nanostructure Integration Techniques for Manufacturable Devices, Circuits, and Systems: Interfaces, Interconnects, and Nanosystems, 60030F (12 November 2005); doi: $10.1117 / 12.632157$

SPIE. Event: Optics East 2005, 2005, Boston, MA, United States 


\title{
A new imaging method: optofluidic microscopy
}

\author{
Xin Heng ${ }^{* 1}$, David Erickson ${ }^{2}$, Demetri Psaltis ${ }^{1}$, Changhuei Yang ${ }^{1}$ \\ ${ }^{1}$ Electrical Engineering, California Institute of Technology, Pasadena, CA, 91125; \\ ${ }^{2}$ Mechanical and Aerospace Engineering, Cornell University, Ithaca, NY, 14853
}

In this work, we present a novel optical imaging device that can be directly integrated into a microfluidic network, and can therefore enable on-chip imaging in a microfluidic system. This micro imaging device, termed optofluidic microscope (OFM) is free of bulk optics and is based on a nanohole array defined in a nontransmissive metallic layer that is patterned onto the floor of the microfluidic channel. The operation of the optofluidic microscope is explained in details and its performance is examined with Caenorhabditis elegans $(C$. elegans) of various genotypes. Images from a large population of worms are efficiently acquired within a short time frame. The quality of the OFM images of $\mathrm{C}$ elegans and the morphological characteristics revealed by the images are evaluated. The experimental results support our claim that the methodology described therein can be effective in a powerful tool for fulfilling high-resolution, high-throughput imaging task of the microscopic biological samples.

Keywords: Nanophotonics, Microfluidics, C elegans phenotyping

\section{Introduction}

There are numerous inherent advantages associated with micro total analysis system ( $\mu \mathrm{TAS}$ ) that uses optical light for probing and sample manipulations. These advantages include the non-destructive nature of optical probing, the ease by which light fields may be reconfigured, and the ability of light to 'walk through' transparent materials to manipulate objects. It is, therefore, not surprising that a lot of efforts have been recently directed towards the incorporation of optical tools into $\mu$ TAS. Optical manipulation tools of this category include microfluidic sorter operated by optical force switching [1], optical tweezers in microfluidics [2], and all-optical microfluidic particle-sorter [3]. At the same time, various miniaturized optical probing and analyzing implementations have been realized with improved performance and reduced economical cost. These include micro fluorescence activated cell sorter [4], back scattering interferometry in rectangular channels (BIRC) [5], and numerous microscopy or fluorescence imaging tools applied to virus [6], DNA molecules [7, 8], single cells [9] and micro-organisms [10].

Although the microfluidic portion of these above mentioned setups is compact and cost effective, the optical components tend to be fairly complicated and occupy large space. There are numerous efforts presently underway to implement systems that seek to incorporate compact optical systems into microfluidics [11-14]. However, there has been little work in the design of a high-resolution optical imaging tool that can be fully integrated onto a microfluidic system. In this article, we present, to our knowledge, the first step in the direction.

\section{Methods}

The device, termed optofluidic microscope (OFM) can be readily miniaturized, and is capable of acquiring highresolution images of biological samples that are flowing in microfluidic channels. High-throughput imaging of individual biological samples can be efficiently acquired with a linear CCD array; the OFM images may then be used to perform phenotype sorting. Caenorhabditis elegans (C. elegans) is chosen as our first test organism to verify our system's capability to perform high-speed imaging and accurate evaluation of samples' morphological features.

The OFM device consists of an elastomeric microfluidic channel that has a clear transmission ceiling and an optically absorptive floor made of metallic material (Fig. 1(a)).

\footnotetext{
* Correspondence and requests for materials should be addressed to Xin Heng (xin@caltech.edu)
} 
A slanted line of transmission holes is etched into the opaque floor. In our prototype, the holes' diameter is about 600 $\mathrm{nm}$, and the optical absorptive layer is made of aluminum or gold and is about $80 \mathrm{~nm}$ thick in either case. Fabrication and alignment are detailed in Supplementary Materials. The device can then be mounted on top of a one dimensional



Figure 1: (a) 3D layout of OFM; (b) view from the bottom: Isolated hole [a] matches with hole [b] in the nanoarray; (c) schematic setup; (d) representative signal time traces from three holes. red (hole \#0): isolated hole, green (hole \#49): its correspondence , blue (hole \#86): un-occluded hole 
CCD or photodiode array. For clarity, we shall define a coordinate system in relation to the O FM device. We set the $\mathrm{x}-$ axis to be along the flow direction and the y-axis to be normal to it (depicted in inset of Fig. 1(a)). Z-direction is normal to the device's plane. If we choose the inter-hole separation along the x-direction to be equal to the CCD's pixel size, the transmission through each hole will map to individual pixel. The determination of an object's shape and profile is accomplished by flowing the object across the line of holes. Notice that the system's pixel size along the y-axis is set by the separation between the holes along $\mathrm{y}$-axis; and that along the $\mathrm{x}$-axis is equal to the ratio of the fluid flow speed and the pixel acquisition rate. The system resolution is by no means limited by the size of the pixels on the CCD array. The staggered-hole-array approach enables us to achieve high resolution imaging $(\sim 600 \mathrm{~nm})$ with existing CCD linear arrays where the pixel size is typically $10 \mu \mathrm{m}$. For the purpose of testing the validity of OFM's working principle, a conventional inverted microscope is used in our experiments (Fig. 1 (c)). Transmission curves of individual holes are projected onto a CCD array (Princeton Instruments Spec-10 100) with the use of an Olympus IX51. The role of the microscope is simply to map each hole transmission uniquely onto individual pixels on the CCD array.

\section{Experimental results}

To demonstrate OFM's ability to perform imaging and phenotype sorting, we decided to focus on one particular application- to perform phenotype identification on a popular animal model- $\mathrm{C}$ elegans. We prepare the samples by first killing them in a heat bath at $70^{\circ} \mathrm{C}$ for 3 minutes. At the end of the process, the worms tend to be relaxed and straightened out. We then mix the worm with a $0.1 \%$ solution of Bovine Serum Albumin (BSA). The BSA solution helps the worms to more easily move in the microchannel. $\mathrm{C}$ elegans are transported through the channel by gravitational force, i.e. the microchip is vertically mounted. As each worm passes with a constant speed through the region that contains the slanted nanohole array, each individual hole will take a fine line scan over the sample in $\mathrm{x}$ direction. The time shift of the signals between adjacent holes will be a generic constant $(\tau)$ and thus can be easily corrected. Therefore we end up with a sequence of transmission time traces through individual nanoholes. To obtain the time-shift constant $\tau$, an isolated hole of the same size is drilled at either end of the nanohole array; the hole along the nanohole array that matches the isolated hole's y-axis displacement is then located (Fig. 1(b)). The time shift

$$
\tau=\frac{\Delta x \times \Delta f r \times \delta t}{\Delta L}
$$

where $\Delta \mathrm{fr}$ is the frame lag between these two correspondent holes when the object flows across them, respectively; $\Delta \mathrm{x}$ is inter-hole separation projected in $\mathrm{x}$ direction; $\delta \mathrm{t}$ is camera's acquisition time (typically $2.5 \mathrm{msec}$ ) and $\Delta \mathrm{L}$ is the separation of those two correspondent holes. The pixel size in $\mathrm{x}$ direction $(\delta \mathrm{x})$ is determined as



(a)

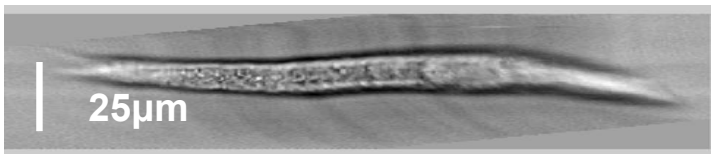

(b)

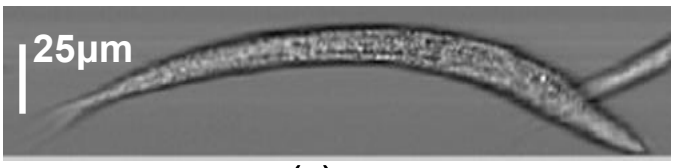

(c)

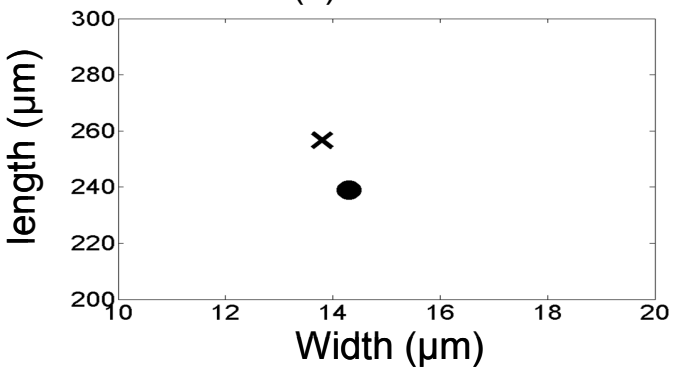

(d)

Figure 2: (a) Conventional microscope image of C. elegans (dpy-24); (b) OFM image of mutant dpy-24; (c) OFM image of wild type (N2); (d) The location of various types of worms on a length-waist map (cross (X): wild-type; dot (.): dpy-24). 
$\delta x=\frac{\Delta x \times \delta t}{\tau}=\frac{\Delta L}{\Delta f r}$

The transmission time changes from three representative nanoholes are presented in Fig.1 (d). The shapes of the signal from two corresponding holes mentioned in the last paragraph are highly similar. It implies that this particular worm passed through the nanohole array without significant rotation. The rotated samples are readily screened out by looking for disparity between the two transmission signal traces.

C elegans is one of the most studied model animals in biology due to its genetic tractability, completely sequenced genome, simple anatomy and body transparency. Studying varied nematode phenotypes has helped scientists to gain better understanding of signal transmission pathway in neurobiology, aging, mating and some other physiological behaviors. However, the parameters that are characterized with current $\mathrm{C}$ elegans research are mostly qualitatively described by adjectives such as 'dumpy', 'small', 'slow', etc. This is because the difference between genotypes often remains subtle to human observers and there is a lack of smart imaging systems that can acquire quantitative data detailing their behavior or morphology in a labour saving manner. The optofluidic microscope can use the state of the art microfluidic technology to swiftly deliver biological organisms through the detection region (a chamber or a portion of fluidic channel) and image them, thus saving scientists a decent amount of time from having to measure one worm after another. For example, we have succeeded in getting data from more than 50 wild type C elegans larvae (L1) in less than 3 minutes. The extremely limited space occupied by fluidic channels (a few microns wide and tall) readily eliminates the potential troubles incurred by background interruption.

OFM's images of two different types of nematodes: wild-type (N2) and genotype dpy-24 are shown in Fig 2 (c) and Fig 2 (b), respectively. Wild type C elegans larva at the stage of L1 has its body length (L) at $256.60 \mu \mathrm{m}$ and its waist (W) at $13.80 \mu \mathrm{m}$, which gives a ratio of skeleton length over waist $(\mathrm{L} / \mathrm{W}$ ) of about 18.6. Dpy-24 (see a micrograph in Fig. 2(a)) has been identified in screens for recessive mutations on chromosome I and this mutation affects body morphology, i.e. animals are expected to be slightly shorter and stouter than wild type (weak dumpy as qualitatively described in www.wormbase.org). Our OFM device successfully rendered a quantitative description of dpy-24 mutant's morphology, i.e. $\mathrm{L}=239.0 \mu \mathrm{m}$, $\mathrm{W}=14.3 \mu \mathrm{m}, \mathrm{L} / \mathrm{W}=16.7$. Fig 2 (d) maps the parameters mentioned above into a length-width graph. More work is presently in progress to analyze all the data we have obtained for these two phenotypes, which will not only offer a more detailed characterization of their growth and development, but also provide a statistical overview of the more subtle variance within the population of the same phenotypes. This inner-group statistical comparison is believed to be significant in studying the effects of environment (e.g. temperature, humidity), nutrition and drugs on the worm entities that have an identical starting point and genotype.

\section{Nanohole transmission}

Besides the experimental verification of the operation of OFM device, some preliminary calculations have been done with the focus on finding out the correlation of the system's ultimate resolution and its

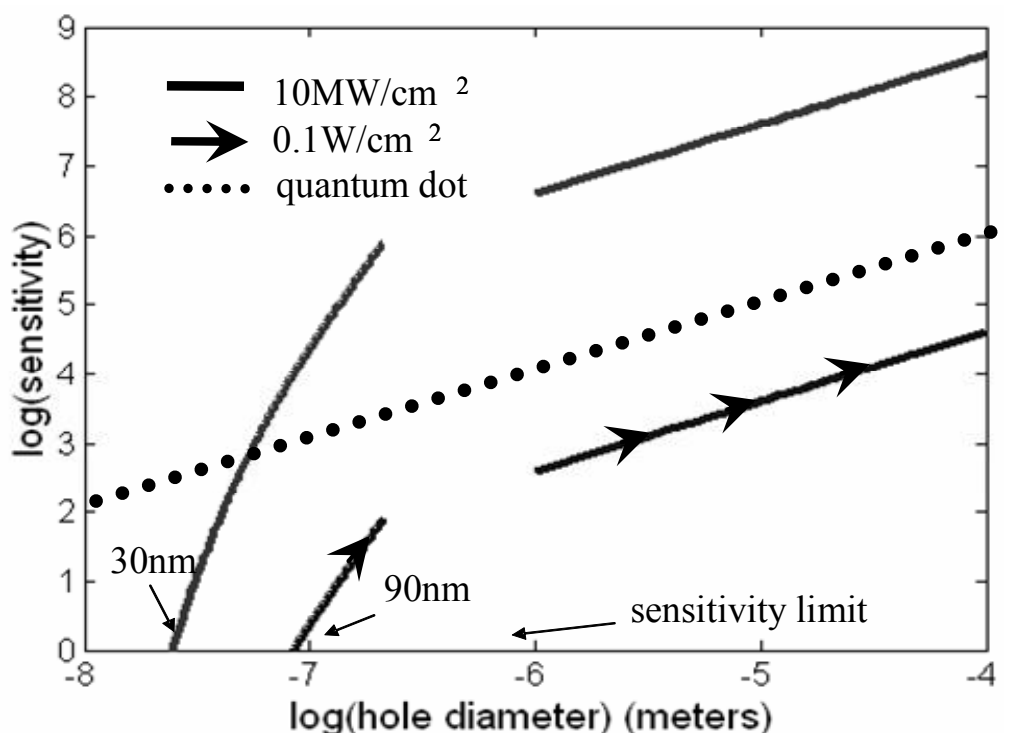

Figure 3: Transmission sensitivity curves for varying hole sizes at two illumination intensity $\left(10 \mathrm{MW} / \mathrm{cm}^{2}\right.$ : biological laser illumination intensity limit, $0.1 \mathrm{~W} / \mathrm{cm}^{2}$ : ambient sunlight intensity). Green line denote the sensitivity curve for an OFM implementation with quantum dots in place of the holes (intensity $\sim 100 \mathrm{~W} / \mathrm{cm}^{2}$ ). This implementation is expected to perform well at high resolution. The sensitivity curves for the holes are derived through analysis in two different size regimes. 
sensitivity. Sensitivity of OFM device depends critically on the total transmission through each hole. Assuming the metal layer is perfectly conductive, two different regimes of hole size $\left(S_{h}\right)$ are examined.

$S_{h}>>\lambda$, Large hole limit. In this regime, the effective transmission area $A_{T}$ is simply equal to the physical cross section of the hole.

$S_{h}<<\lambda$, Small hole limit. In this regime and in the limit that the hole is infinitesimally thin, Bethe [15] showed that the effective transmission area is given is proportional to the sixth power of the pinhole diameter. In a recent work, De Abajo [16] observed that the transmission is further attenuated exponentially as a function of the hole depth. Combining these two effects, we postulate that the effective transmission area can be expressed as:

$$
A_{T}=\left(\frac{16 \pi^{3}}{27}\right)\left(\frac{S_{h}^{6}}{\lambda^{4}}\right) \exp \left(-4 \pi d \sqrt{\frac{0.586^{2}}{S_{h}{ }^{2}}-\frac{1}{\lambda^{2}}}\right)
$$

The formulation agrees well with the simulation data that De Abajo [16] reported. The total transmission photon count for a pixel dwell time $\tau$ (also equivalent to the inverse of frame rate) is given by,

$$
N_{T}=\frac{\varepsilon I A_{T} \tau}{h \frac{c}{\lambda}}
$$

where $h \frac{c}{\lambda}$ : the energy that one single photon carries; $I:$ illumination intensity; $\varepsilon:$ quantum efficiency of CCD camera.

The dominating noise source includes the photon counting noise (shot noise) and the receiver noise $\left(n_{r} \tau\right)$. Thus the sensitivity (SNR, or signal to noise ratio) can be expressed as:

$$
S N R=\frac{N_{T}}{\sqrt{N_{T}+\left(n_{r} \tau\right)^{2}}}
$$

Therefore, object imaging with a micron level resolution and $30 \mathrm{~dB}$ sensitivity can be readily performed with the use of natural light illuminations. In principal, sub-wavelength resolution can be achieved in an OFM device by simply spacing the adjacent holes in the y-direction at the desired resolution limit. As the holes are well separated in xdirection, their transmission contributions will be distinguishable from each other on CCD camera. The state of the art nanofabrication technology enables the creation of etching patterns with resolution of 10's of nanometer. Therefore, it should be possible to create OFM devices with resolution of sub 100 nanometers. Our preliminary estimates based on Equations (1)-(3) shown in graphical forms in Fig. 3 predicts that with $600 \mathrm{~nm}$ laser illumination, we can easily achieve $40 \mathrm{~dB}$ signal sensitivity for OFM with resolution of $100 \mathrm{~nm}$.

\section{Conclusion}

We have presented what to our knowledge is the first effort at integrating a microscope into a single biochip. We have demonstrated that OFM holds much promise of being a reliable and efficient on-chip microscopy tool with high resolution. The images OFM from $\mathrm{C}$ elegans of different phenotypes show well distinguishable morphological features that were time-consuming and difficult to obtain by direct human observation. OFM can be used as a high-accuracy phenotyping device in the research of neurobiology, development and behavior. 


\section{Supplementary Materials}

\section{Chip fabrication and alignment:}

The nanohole array is defined in non-transmissive metal foil, i.e. gold or aluminium. In the fabrication of nano array in gold, holes are directly made by focused ion beam (FIB) on a 80nm thick gold film, which is deposited on ultra-clean quartz wafer with chromium as the adhesion layer material; in the fabrication of nano array in aluminium, we deposit a 90-nanometer layer of $\mathrm{Al}$ on clean quartz wafer followed by spinning a 200nm layer of PMMA on top of it. Next, the nanohole array is patterned on PMMA by using high resolution electron beam writer, and later transferred into aluminium by using dry ion beam etching. In the prototype used in this demonstration experiments, the diameter of the nanohole is all about 600nm. The single-layer PDMS microfluidic chip is produced by standard replica molding. In light of the present application purpose, the channel cross section is about $30 \mu \mathrm{m}$ wide and $20 \mu \mathrm{m}$ tall. The alignment of the fluidic layer and the metallic layer is accomplished by using an inverted microscope or a Suss mask aligner. Afterwards, the alignment can be verified by using an upright microscope.

\section{Acknowledgement}

We are grateful for the generous help from Dr. Larry R. Baugh and Professor Sternberg regarding C elegans culturing and phenotyping, and from Professor Axel Scherer, Dr. Joerg Schilling in fabrication. We thank Dr. Zahid Yaqoob, Kevin Reynolds, Xiquan Cui for constructive discussions. The assistance from Cornell Nanofabrication Facility and Caltech Watson cleanroom is well appreciated. This project is funded by DARPA's center for optofluidic integration

\section{Reference}

1. Wang, M.M., et al., Microfluidic sorting of mammalian cells by optical force switching. Nature Biotechnology, 2005. 23(1): p. 83-87.

2. Grier, D.G., A revolution in optical manipulation. Nature, 2003. 424(6950): p. 810-816.

3. MacDonald, M.P., G.C. Spalding, and K. Dholakia, Microfluidic sorting in an optical lattice. Nature, 2003. 426(6965): p. 421-424.

4. Fu, A.Y., et al., A microfabricated fluorescence-activated cell sorter. Nature Biotechnology, 1999. 17(11): p. 11091111.

5. Markov, D.A., K. Swinney, and D.J. Bornhop, Label-free molecular interaction determinations with nanoscale interferometry. Journal of the American Chemical Society, 2004. 126(50): p. 16659-16664.

6. Akin, D., H.B. Li, and R. Bashir, Real-time virus trapping and fluorescent imaging in microfluidic devices. Nano Letters, 2004. 4(2): p. 257-259.

7. Mangenot, S., et al., Real-time imaging of DNA ejection from single phage particles. Current Biology, 2005. 15(5): p. $430-435$.

8. Tegenfeldt, J.O., Near-field scanner for moving molecules. Physical review letters, 2001. 86(7): p. $1378-1381$.

9. Li, P.C.H., et al., Transport, retention and fluorescent measurement of single biological cells studied in microfluidic chips. Lab on a Chip, 2004. 4(3): p. 174-180. 
10. Lange, D., et al., A microfluidic shadow imaging system for the study of the nematode Caenorhabditis elegans in space. Sensors and Actuators B-Chemical, 2005. 107(2): p. 904-914.

11. Koschwanez, J., et al., Identification of budding yeast using a fiber-optic imaging bundle. Review of Scientific Instruments, 2004. 75(5): p. 1363-1365.

12. Ruano, J.M., et al., Flame hydrolysis deposition of glass on silicon for the integration of optical and microfluidic devices. Analytical Chemistry, 2000. 72(5): p. 1093-1097.

13. Valentino, J.P., S.M. Troian, and S. Wagner, Microfluidic detection and analysis by integration of thermocapillary actuation with a thin-film optical waveguide. Applied Physics Letters, 2005. 86(18).

14. Zhang, D.Y., N. Justis, and Y.H. Lo, Integrated fluidic lenses and optic systems. IEEE Journal of Selected Topics in Quantum Electronics, 2005. 11(1): p. 97-106.

15. Bethe HA, Theory of diffraction by small holes, physics Review 1944, 66, p163.

16. de Abajo F., 'Light transmission through a single cylindrical hole in a metallic film', Optics Express, 2002,10 , p. 1475-1484. 